\title{
MAXIMUM GRAPHS NON-HAMILTONIAN-CONNECTED FROM A VERTEX
}

\author{
by G. R. T. HENDRY
}

(Received 30 September, 1982)

A path (cycle) in a graph $G$ is called a hamiltonian path (cycle) of $G$ if it contains every vertex of $G$. A graph is hamiltonian if it contains a hamiltonian cycle. A graph $G$ is hamiltonian-connected if it contains a $u-v$ hamiltonian path for each pair $u, v$ of distinct vertices of $G$. A graph $G$ is hamiltonian-connected from a vertex $v$ of $G$ if $G$ contains a $v-w$ hamiltonian path for each vertex $w \neq v$. Considering only graphs of order at least 3 , the class of graphs hamiltonian-connected from a vertex properly contains the class of hamiltonian-connected graphs and is properly contained in the class of hamiltonian graphs.

Our present object is to determine the maximum possible number of edges in a graph of order $p \geqslant 3$ which is not hamiltonian-connected from a vertex and to describe the graphs which attain this maximum. The proof of Theorem 1 leans heavily on the following 3 results (Theorems $\mathrm{A}$ and $\mathrm{B}$ are due to Ore and Bondy and Theorem $\mathrm{C}$ is due to Chartrand and Nordhaus).

THEOREM A. If $G$ is a non-hamiltonian graph of order $p \geqslant 3$, then (i) $|E(G)| \leqslant$ $\frac{1}{2}\left(p^{2}-3 p+4\right)$ and (ii) if equality holds in (i) then $G$ is $K_{2}+\bar{K}_{3}$ or, for $p \geqslant 3, K_{2} . K_{p-1}$ (i.e. the graph of order $p$ having 2 blocks: $K_{2}$ and $\left.K_{p-1}\right)$.

THEOREM B. If $G$ is a non-hamiltonian-connected graph of order $p \geqslant 4$ then (i) $|E(G)| \leqslant \frac{1}{2}\left(p^{2}-3 p+6\right)$ and (ii) if equality holds in (i) then $G$ is $K_{6}-K_{3}$ or, for $p \geqslant 4$, $K_{\mathrm{p}}-K_{1, \mathrm{p}-3}$.

TheOREM C. Let $G$ be a hamiltonian graph of order $p$. If there exists a vertex $u$ such that

$$
\operatorname{deg}(u)+\operatorname{deg}(v) \geqslant p+1
$$

for each vertex $v$ not adjacent to $u$, then $G$ is hamiltonian-connected from at least 2 vertices.

THEOREM 1. If $G$ is a graph of order $p \geqslant 3$ which is not hamiltonian-connected from $a$ vertex then (i) $|E(G)| \leqslant \frac{1}{2}\left(p^{2}-3 p+4\right)$ and (ii) if equality holds in (i) then $G$ is $C_{4}, K_{2}+\bar{K}_{3}$ or, for $p \geqslant 3, K_{2} \cdot K_{\mathrm{p}-1}$.

Proof. If $p=3$ the result is obvious; therefore assume $p \geqslant 4$.

(i) Since $G$ is not hamiltonian-connected from a vertex, it is not hamiltonianconnected and so, by Theorem $\mathrm{B}(\mathrm{i}),|E(G)| \leqslant \frac{1}{2}\left(p^{2}-3 p+6\right)$. If $|E(G)|=\frac{1}{2}\left(p^{2}-3 p+6\right)$ then, by Theorem B(ii), $G$ is either $K_{6}-K_{3}$ or, for $p \geqslant 4, K_{\mathrm{p}}-K_{1, p-3}$. But neither of these is possible since $K_{6}-K_{3}$ is hamiltonian-connected from any of its vertices of degree 3 and $K_{p}-K_{1, p-3}$ is hamiltonian-connected from the vertex at the centre of the $K_{1, p-3}$. Therefore, $|E(G)| \leqslant \frac{1}{2}\left(p^{2}-3 p+4\right)$.

Glasgow Math. J. 25 (1984) 97-98. 
(ii) Now suppose that $G$ has exactly $\frac{1}{2}\left(p^{2}-3 p+4\right)$ edges. If $G$ is not hamiltonian then, by Theorem A(ii), $G$ is either $K_{2}+\bar{K}_{3}$ or, for $p \geqslant 4, K_{2} \cdot K_{\mathrm{p}-1}$. From now on suppose that $G$ is hamiltonian. If $p=4$ then clearly $G$ must be $C_{4}$. Therefore, suppose $p \geqslant 5$ and that $v_{1}, v_{2}, \ldots, v_{p}, v_{1}$ is a hamiltonian cycle of $G$. If $G$ has a vertex, say $v_{1}$, of degree $p-1$ then $G$ is hamiltonian-connected from both $v_{2}$ and $v_{\mathrm{p}}$ which is a contradiction. However, $G$ must have a vertex of degree $p-2$ since, if it doesn't, then it has at most $\frac{1}{2}\left(p^{2}-3 p\right)<$ $\frac{1}{2}\left(p^{2}-3 p+4\right)$ edges. Suppose, without loss of generality, that $\operatorname{deg}\left(v_{1}\right)=p-2$ and that $v_{i}$ is the vertex not adjacent to $v_{1}$. If $\operatorname{deg}\left(v_{j}\right) \geqslant 3$ then it follows from Theorem $\mathrm{C}$ that $G$ is hamiltonian-connected from at least 2 vertices which is a contradiction. Therefore, $\operatorname{deg}\left(v_{\mathrm{i}}\right)=2$ and we have

$$
\begin{aligned}
\frac{1}{2}\left(p^{2}-3 p+4\right) & =|E(G)| \\
& =\frac{1}{2} \sum_{i} \operatorname{deg}\left(v_{i}\right) \\
& \leqslant \frac{1}{2}(2+(p-1)(p-2)) \\
& =\frac{1}{2}\left(p^{2}-3 p+4\right) .
\end{aligned}
$$

Equality must hold throughout so that every vertex of $G$, except $v_{i}$, has degree $p-2$. In particular, $\operatorname{deg}\left(v_{j-1}\right)=p-2$ and if $v_{k}$ is the single vertex not adjacent to $v_{j-1}$ then, since $k \neq j, \operatorname{deg}\left(v_{k}\right)=p-2$. If $2 p-4=\operatorname{deg}\left(v_{j-1}\right)+\operatorname{deg}\left(v_{k}\right) \geqslant p+1$ then, by Theorem $\mathrm{C}, \mathrm{G}$ is hamiltonian-connected from at least 2 vertices. Therefore, $2 p-4<p+1$, i.e. $p<5$. This is a contradiction which completes the proof.

\section{REFERENCES}

1. J. A. Bondy, Variations on the Hamiltonian theme, Canad. Math. Bull. 15 (1972), 57-62.

2. G. Chartrand and E. A. Nordhaus, Graphs Hamiltonian-connected from a vertex, The theory and applications of graphs, edited by G. Chartrand et al. (John Wiley, 1981), 189-201.

3. O. Ore, Hamilton connected graphs, J. Math. Pures Appl. 42 (1963), 21-27.

4. O. Ore, Note on Hamilton circuits, Amer. Math. Monthly 67 (1960), 55.

\section{UNIVERSITY OF ABERDEEN}

Present address:

12 Cliff Terrace

BUCKIE

BANFFSHIRE

SCOTLAND

AB5 1LX 